\title{
Instituting Pandemic Plans in a Singapore Anaesthesia Unit: The Challenges and Learning Points
}

\author{
Lydia Weiling Li ${ }^{*}$, Prit Anand Singh1\#, Chou Liang Mah¹, Ong Lay Teng², Qian Jun Tong1, \\ Xiang Long Louis Ng 1
}

${ }^{1}$ Department of Anaesthesia and Surgical Intensive Care, Changi General Hospital, Singapore

${ }^{2}$ Division of Nursing, Operating Theatre, Changi General Hospital, Singapore

Email: *lydia.li.w@singhealth.com.sg

How to cite this paper: Li, L.W., Singh, P.A., Mah, C.L., Teng, O.L., Tong, Q.J. and Ng, X.L.L. (2020) Instituting Pandemic Plans in a Singapore Anaesthesia Unit: The Challenges and Learning Points. Open Journal of Anesthesiology, 10, 263-276. https://doi.org/10.4236/ojanes.2020.107023

Received: June 24, 2020

Accepted: July 21, 2020

Published: July 24, 2020

Copyright $\odot 2020$ by author(s) and Scientific Research Publishing Inc. This work is licensed under the Creative Commons Attribution International License (CC BY 4.0).

http://creativecommons.org/licenses/by/4.0/

\begin{abstract}
The World Health Organization declared COVID-19 as a pandemic on 11 March 2020. Its rapid spread has put a strain on healthcare systems globally. Singapore ranked the highest in terms of reported cases outside of China in the first few weeks of this outbreak. The management of a patient with COVID-19 in the Operating Theatre (OT) presents a unique set of challenges to the Anaesthetist. Delivery of timely and quality care must be upheld while reducing the risk of transmission to healthcare staff and other patients. This article describes our Anaesthesia Unit's experiences and challenges in instituting our pandemic plans. The authors hope that the sharing of our experience and practical approach would be useful to other Anaesthesia Units worldwide.
\end{abstract}

\section{Keywords}

COVID-19, Anaesthesia, Operation Theatre, Preparedness, SARS-CoV-2, Pandemic, PPE, Manpower

\section{Introduction \& Background}

From the time the first case of COVID-19 was reported in Wuhan, China in December 2019, there has been an exponential rise in the number of COVID-19 cases reported worldwide with an alarming increase in the number of mortalities [1] [2]. Singapore was the third country outside China to have confirmed cases of COVID-19 and ranked amongst the highest in terms of reported cases in the first few weeks of the outbreak [3]. At the time of writing, more than 200 countries have been affected, with over 600,000 confirmed cases and more than \#Co-author. 
28,000 deaths across different continents [3]. The World Health Organization declared COVID-19 as a pandemic on 11 March 2020 [4]. As a result of the rapid spread of the COVID-19 virus, healthcare systems across the world have come under increasing strain to cope with the burden of disease on top of caring for the non-COVID-19 related patients. Concomitantly medical personnel have been under constant threat to the risk of infections due to the highly contagious nature of the disease as well as severe shortages of Personal Protective Equipment (PPE) at the frontlines [5].

The management of a patient with COVID-19 in the Operating Theatre (OT) presents a unique set of challenges. The Anaesthetist is often at the highest risk of exposure due to involvement in aerosol generating procedures (AGP).

Changi General Hospital (CGH) is a 1000-bed public hospital providing a range of advanced medical care in the Eastern part of Singapore. It serves a population of about 1 million residents and is the designated hospital providing tertiary care to the local prison population.

In anticipation of the COVID-19 case surge, our institution and department preemptively implemented containment and risk mitigation strategies at various levels based on existing pandemic plans, guidelines and best practices [6]-[12]. The core philosophy was the sustainable deployment of limited manpower and logistical resources in what would likely be a protracted pandemic, while ensuring proactive adaptation of strategies to a rapidly evolving situation.

In this article, we use a case vignette to highlight our dynamic response and adaptation of unit strategies to the practical challenges faced (Table 1).

\section{Case Vignette}

A few days before Singapore's first confirmed report of community transmission and nationwide escalation of infection control and containment measures in February 2020, a 70-year-old patient from China presented at our Emergency Department (ED) after office hours with per-rectal bleeding. Within minutes of arrival, the patient had an episode of massive hematemesis leading to cardiovascular collapse. The Code Blue Team was activated and Cardio-Pulmonary-Resuscitation (CPR) was commenced immediately. Rhythm analysis showed Pulseless Electrical Activity (PEA). Presumptive cause of collapse was hypovolemia and Massive Transfusion Protocol (MTP) was initiated. Spontaneous circulation was restored within a minute. She was rushed into the Emergency Operation Theatre (EOT) for urgent Oesophago-Gastro-Duodenoscopy (OGD) keeping in view a need for laparotomy for hemostasis.

Although the patient did not fit the case definition at admission for having COVID-19, the on call Anaesthetic and Surgical team made a joint decision to treat the patient as a suspected COVID-19 case due to the urgency of operation and the potential of asymptomatic carriers from China. This decision implied that all personnel involved in patient care would have to be in full PPE. This included the donning of Powered-Air Purifying Respirator (PAPR) (3M-Jupiter), N95 mask, face shield/goggles, protective splash-proof gown and gloves. 
Table 1. Summary of challenges faced and practical modifications taken.

\begin{tabular}{|c|c|}
\hline Challenges Faced & Modifications/Comments \\
\hline $\begin{array}{l}\text { Uncertainty in risk stratification } \\
\text { of patients due to evolving case } \\
\text { definitions }\end{array}$ & $\begin{array}{l}\text { - Institution set up a Pandemic Response Planning Team } \\
\text { - Formation of dedicated Department workgroup to oversee Pandemic Response } \\
\text { - Updated case definitions based on national and global situation } \\
\text { - Recognition that case definitions will always lag behind actual cases } \\
\text { - Anaesthetist should and must take on an active leadership role in risk assessment of all patients } \\
\text { presenting for surgery } \\
\text { - Creation of user-friendly, quick screening questionnaire for intra-Department usage (see Figure 1) }\end{array}$ \\
\hline $\begin{array}{l}\text { Timely dissemination of } \\
\text { information }\end{array}$ & $\begin{array}{l}\text { - Establishment of effective communication channels } \\
\diamond \text { Detailed information disseminated through work emails } \\
\diamond \text { Practical summaries disseminated real-time through encrypted mobile messaging systems }\end{array}$ \\
\hline Manpower rostering & $\begin{array}{l}\text { - Uncertainty of evolving global situation and need for preparedness for a surge in patients } \\
\text { - Prohibition of overseas leave for all staff to augment manpower } \\
\text { - OT Team segregated into } 2 \text { teams with each team working } 7 \text { continuous days and } 12 \mathrm{~h} \text { shifts to } \\
\text { minimise risk of cross-transmission } \\
\text { - Simulation of contingency roster for } 3 \text { weeks to ensure workability, then down-triaging to routine roster } \\
\text { to prevent burnout } \\
\text { - Use of shadow roster-routine roster ongoing with capability to implement contingency roster almost } \\
\text { immediately should the need arise }\end{array}$ \\
\hline Unfamiliarity with PAPR & $\begin{array}{l}\text { - Staff education } \\
\diamond \text { Regular refresher sessions for Anaesthetists and Nurses to increase familiarity with PAPR } \\
\diamond \text { Donning and removing of PAPR to improve staff's response time } \\
\diamond \text { In situ Simulation training including resuscitation scenarios with the use of PAPR }\end{array}$ \\
\hline $\begin{array}{l}\text { Communication difficulties } \\
\text { with PAPR }\end{array}$ & $\begin{array}{l}\text { - Instructions to be brief and succinct } \\
\text { - Effort made to speak clearly and project your voice } \\
\text { - Acknowledgement and confirmation via read back and hand sign } \\
\text { - Maintain situational awareness and alertness }\end{array}$ \\
\hline Sustainability of supply of PPE & $\begin{array}{l}\text { - Centralised distribution to ensure close monitoring of supplies } \\
\text { - Diversification of procurement and establishment of reliable supply chain in advance }\end{array}$ \\
\hline Healthcare worker fatigue & $\begin{array}{l}\text { - Clear directives from Pandemic Preparedness Committee } \\
\text { - Reliable flow of communication to allay anxieties } \\
\text { - Allowance of rest days for staff while remaining contactable and on stand-by } \\
\text { - Anonymous hotlines for peer support or counselling services made available for all staff }\end{array}$ \\
\hline
\end{tabular}

Team huddle was performed prior to the arrival of the patient to EOT. It involved the Anaesthesia Consultant, Anaesthesia Residents, Surgeons, Scrub and Anaesthesia Nurses. This allowed all members of the team to be on board with the plans to ensure seamless delivery of quality care. Moreover, the requisite equipment such as Level $1^{\mathrm{TM}}$ Rapid Infusor system and surgical equipment could be preemptively prepared. This minimised any unnecessary movement in and out of the OT during the case, reducing delays and possible risk of cross contamination.

Manpower was reduced to only those necessary to minimise exposure-the Anaesthesia Consultant, one junior Resident and one Anaesthesia Nurse. The remaining anaesthesia resident and other anaesthesia nurses would function as runners stationed in the attached induction room where they could be easily called upon at short notice. The most direct route to EOT was planned in advance with the patient bypassing the induction room to minimise contamination 
of the OT Complex.

The patient arrived hypotensive and tachycardic with ongoing massive blood transfusion. She was induced via Rapid Sequence Induction and intubated with a Video laryngoscope by the Anaesthesia Consultant on first attempt. Laparotomy was performed as surgeons were unable to locate the source of bleeding for haemostasis during OGD. Anterior gastrotomy was performed as the gastric antrum was identified as the most likely area of bleeding. This however did not lead to significant haemostasis. Upon further exploration, the source of bleeding was found to be at the posterior of proximal duodenum and a duodenotomy was performed with successful haemostasis.

The patient was transferred to the Surgical Intensive Care Unit (SICU) intubated postoperatively for hemodynamic monitoring after further resuscitation in EOT. Clear communication was established early with our colleagues in SICU and arrangements were made to segregate the suspected case with a designated nursing staff to minimise the risk of cross-transmission/contamination to other patients/areas/staff in the Unit.

Prior to transfer and switching of patient from anaesthetic machine to transport ventilator, patient was given fractional inspired oxygen of 1.0, adequately re-paralysed (Train-of-Four guided) and anaesthetised with intravenous agents. We modified our airway transfer technique to ensure the patient's breathing circuit was maintained as a closed system throughout the process. This was done through the use of an artery forceps to clamp the endotracheal tube while leaving the Heat \& Moisture Exchange Hepa Filter (HME) on and ensuring the ventilator was switched off prior to any disconnection from the circuit. The unclamping of the endotracheal tube was only done after the re-establishment of a closed circuit. The aim was to ensure minimal aerosolization of patients' secretions into the environment. These precautions were repeated in the SICU. The route taken to the SICU was also pre-planned to minimise environmental contamination.

Extended time was given for a thorough cleaning and decontamination of the EOT as per institutional guidelines, as discussed in section below.

\section{Discussion}

\subsection{Risk Stratification of Patients}

The challenge in managing COVID-19 patients starts with recognising the infected patient. This uncertainty is compounded in emergency surgeries, where detailed screening may not be possible as illustrated in the case vignette. Decisions as to the level of precautions needed must often be made expediently. In the event of extensive community spread, a decision for full PPE for all patient contact is a given. However, in a situation where community spread is limited, considerations have to be taken to conserve limited resources of PPE. Any decision taken should be weighed and jointly agreed in a coordinated and rapid manner.

The Anaesthetist must take on the leadership role in risk stratification of all 
patients presenting for elective or emergency surgery. As illustrated in the case vignette, our patient straddled the period between the first reported imported case and the first confirmed case of community spread in Singapore. The case definition at that point of time aided by healthy clinical suspicion was adequate to trigger the need for full precaution but the threshold would have been much lower barely a week later when the situation escalated in Singapore.

Up to date knowledge of case definitions will aid care teams in making the correct call for the level of precautions to take. It is a fine balance of risking wasting precious resources versus risking exposing our healthcare workers (HCW) to the disease. The decision on level of precaution should give significant consideration to the fact that HCW protection is of paramount importance as we cannot easily replace the specialist workforce.

To aid in this decision-making process, our Department came up with a user-friendly, quick screening questionnaire for intra-Department usage (see Figure 1).

Close and Updated Communications

To facilitate reliable and timely flow of information, communication channels via encrypted mobile apps were established within the department. Detailed information was sent via work emails daily, while a summary of real-time and practical points were sent out through an encrypted mobile messaging system. This enabled the On-call Anaesthetist/s to be updated in a beat to beat fashion which is critical as most Anaesthesia Units worldwide during an outbreak will work on a roster system and will have minimal face-to-face interactions for exchange of information.

\subsection{Practical Considerations for Conduct of Anaesthesia}

\section{Airway Management}

Safe and competent airway management is the cornerstone for provision of quality General Anaesthesia. However, airway management leads to aerosol generation which is of particular concern in the current COVID-19 outbreak.

Have you returned from overseas in the laast 14 days? If so please state the country/countries visited.*

Do you have flu-like symptoms (e.g. fever, cough, sorethroat, etc.) ?

Did you come into contact with someone who is a suspected or confirmed case of COVID-19 (2019-nCoV)?

Are you currently serving a Home Quarantine Order (HQO) or a Stay Home Notice (SHN)?

*For patient with any. travel history or if 'yes' to questions 2 to 4, please notify listing surgeon

- If indication for surgery is non-urgent: to cancel case

- If indication for surgery is urgent: to discuss case with anaesthetist on proper precautions and post op disposition

Figure 1. Our department's quick screening questionnaire. 
The guiding principle in airway management is to minimise aerosol generation. When managing airways, full precautions are needed with requisite PPE and PAPR. As illustrated in our case vignette, this was achieved at several levels. Firstly, in terms of personnel, airway management was done by the most senior anaesthetist to maximise chances of first attempt success, with help of a video laryngoscope. Secondly, rapid sequence induction was performed to remove the need for ventilating the patient which may disperse aerosol into the surroundings. In terms of equipment, we ensured there was a tight fit of the mask on the patient's face to reduce air leak; Bag Mask Ventilation (BMV) was actively discouraged. 2 HME filters were employed, one at the ETT end and the other at the inspiratory arm of the circle circuit. ETT clamping as described above was also added into our workflow. This practice has become the standard operating procedure for all at risk cases in our OT. As the risk of aerosolized secretions with the use of high flow nasal oxygenation (HFNO) was unclear, our department temporarily advised against its use. Since then, a study by Loh et al. [13] has shown that its use is associated with a larger area of spread, and is not advisable in patients with COVID-19.

As extubation is also a risk for aerosolization of infectious droplets, our department stipulated that all extubations including Laryngeal Mask Airway (LMA) removal would be performed in OT instead of the usual Post Anaesthesia Care Unit (PACU) to minimise risk of contamination. Steps adapting the use of a large plastic bag to cover the face and airway during removal of LMA and ETT have also been practised.

\section{Communications}

In the illustrated case, the teams involved realised that communicating through N95 and PAPR was a challenge. During debriefing, a few guidelines were set when communicating in PAPR within teams. Communications would be short and concise with key words, read back or acknowledgement with hand signals especially for important messages. Communications with the runner outside OT were to be done on white boards and through windows at the OT door. Runners outside would also acknowledge with hand signs or "write back" on another whiteboard to confirm contents of message.

Emergency Airway Service and Cardiopulmonary resuscitation

The anaesthetic team posted in theatres is part of the Emergency Airway Service (EAS) and Code Blue (Resuscitation) callouts. The EAS started in the last decade to help colleagues in other areas (Accident \& Emergency (A\&E), Medical ICU (MICU)), to intubate anticipated/unanticipated difficult intubation of patients who were critically ill. This service gets approximately 3 - 5 patients per month. During the COVID-19 outbreak, there were numerous call outs for intubation and resuscitation of critically ill patients. We recognised that the donning of PPE could result in delay to attend to a patient in a resuscitation situation. However, staff protection was of paramount importance and could not be compromised. We adopted a workflow (Table 2) for resuscitations of high risk 
Table 2. Resuscitation protocol of high risk or SARS-CoV-2 positive patients.

\begin{tabular}{|c|c|c|}
\hline $\begin{array}{l}\text { RESPONSE TEAM } 1 \text { PPE: N95, } \\
\text { GOGGLES, GOWN, GLOVES }\end{array}$ & $\begin{array}{l}\text { RESPONSE TEAM } 2 \\
\text { PPE: AS PER TEAM 1, BUT IN ADDITION } \\
\text { PAPR }\end{array}$ & $\begin{array}{l}\text { RESPONSE TEAM } 1 \\
\text { PPE: NOW AUGMENTED }\end{array}$ \\
\hline $1 \times$ Nurse to respond to patient & $1 \times$ Doctor & \\
\hline $\begin{array}{l}1 \times \text { Nurse to push defibrillator and } \\
\text { resuscitation cart }\end{array}$ & $\begin{array}{l}1 \times \text { Respiratory Therapist } \\
2 \times \text { Nurses }\end{array}$ & $\begin{array}{l}\text { Both nurses will exit resuscitation area } \\
\text { after handing over. }\end{array}$ \\
\hline $\begin{array}{l}\text { Activities: } 100 \% \mathrm{O}_{2} \\
\text { Face Mask/Ventilator + Basic BCLS }\end{array}$ & $\begin{array}{l}\text { On activation to start donning PPE including } \\
\text { PAPR }\end{array}$ & $\begin{array}{l}1 \times \text { nurse will don Full PPE and PAPR } \\
\text { and re-enter to assist in resuscitation } \\
\text { efforts. }\end{array}$ \\
\hline $\begin{array}{l}\text { including attachment of defibrillation } \\
\text { pads }+/- \text { initial shock and other } \\
\text { monitoring Devices }\end{array}$ & $\begin{array}{l}\text { Buddies to ensure adequate protection before } \\
\text { team enters resuscitation area and take over } \\
\text { care from Response Team } 1\end{array}$ & $\begin{array}{l}1 \times \text { nurse will change to PPE and stay } \\
\text { outside resuscitation area and act as } \\
\text { runner }\end{array}$ \\
\hline $\begin{array}{l}{ }^{*} \text { Not to engage in Aerosol Generating } \\
\text { Procedures }\end{array}$ & $\begin{array}{l}\text { Starts ACLS/ATLS procedure including } \\
\text { tracheal intubation }\end{array}$ & \\
\hline
\end{tabular}

patients originally developed by our MICU colleagues. In adopting this workflow, the ICU teams rationalised the interventions that could be allowed to afford adequate staff protection before the full suite of PPE was worn. It was decided that CPR would proceed without mask ventilation to minimise aerosol generation by the initial response team before arrival of the second response team in PAPR, as some ventilation could be achieved through chest compressions with high flow oxygen delivered via a non-rebreather mask. For sole intubation calls, the anaesthetic team wore the requisite PPE before intubation whilst the primary team helped oxygenate the patient. However, there was a miscommunication during one of the call outs which led to exposure of the anaesthesia and MICU team [14].

\subsection{Establishment of Effective Chain of Command and Control in OT}

\section{Manpower}

Our Department formed a dedicated workgroup, supplementing the hospital's pandemic response workgroup and to cater to the unique needs of our clinical duties. Our Department of Anaesthesia and Surgical Intensive Care has 34 Anaesthesia Consultants and 27 Anaesthesia Senior and Junior residents. We cover a wide range of duties, providing specialist staff for the Surgical Intensive Care Unit (SICU) and liaison service, Anaesthesia and Acute \& Chronic Pain Service, Pre-Anaesthetic Assessment Clinic and many other clinical and non-clinical support services.

In anticipation of a surge in SICU patients, a large proportion of our team was allocated and segregated to SICU including the more senior residents as we expected more complex cases in SICU. Clinicians allocated to SICU were not assigned OT duties to prevent cross infection between clinical areas. As such, 18 anaesthesia consultants and 17 junior residents were left for OT services. We 
further segregated our OT Team into 2 teams, with each team working for 7 continuous days and $12 \mathrm{~h}$ shifts. Segregation of staff was aimed at reducing chance of cross contamination of teams and to allow for adequate "wash-out" time to detect onset of symptoms. Although this was less than 14 days incubation period, it was the best option due to manpower constraints. The need to plan for unexpected exposure and infected HCW was emphasized in our contact with the illustrated case vignette.

To augment our manpower, all part-time staff were rostered for full time work in the contingency roster. All new leave applications were prohibited and staff on pre-approved leave were expected to be contactable and recallable at all times. Overseas travel was strongly discouraged with travel to affected regions like China prohibited. To enhance compliance to reduce financial penalties due to such circumstances, our institution and Singapore government took proactive steps to assist HCW in seeking refunds from cancelled trips and took unprecedented steps to compensate HCW who could not get refunds.

\section{OT Reorganisation to cater for surge in patients}

Hospital wide elective operative admissions were reduced to only trauma/emergency and urgent cancer cases as part of our emergency planning to create capacity to respond to the surge in COVID-19 cases.

Our OT complex does not have any negative pressure capable OT. Unfortunately, the high flow and positive pressurised OT creates a risk of disseminating aerosolised contaminants from managing a patient with COVID-19.

Our OT complex has two wings; Main Operating Theatre wing (MOT) for major surgeries and a smaller Day Surgery Operating Theatre wing (DSOT) separated from MOT by a fire door as well as non-interconnected ventilatory systems. DSOT wing was designated for all COVID-19 cases as our experience from the case vignette showed that the proximity of Emergency MOT and the shared ventilatory system within MOT posed too much risk and disruption to the safe usage of other MOTs for normal caseloads and emergency cases.

Two DSOT were set up with essential equipment and supplies for confirmed/suspected COVID-19 to minimise equipment contamination. As there is no adjoining induction room for DSOTs, donning of PAPR and PPE was to be performed in adjacent DSOT with a supervising nurse runner. Removal of contaminated PPE was to be done in en-suite disposal rooms supervised by a second designated nurse in PPE. In our illustrated case, manpower in OT was reduced to only essential staff to reduce risk of exposure. All staff in the designated COVID-19 theatre wore full designated PPE (see Table 3 for details). This was to reduce exposure of manpower from aerosol generating procedures.

In our case vignette, patient transfer was conducted via the most expedient route. This was to minimise contamination of the environment especially by a non-intubated patient.

We refined our Pandemic Planning for OT with some changes. Awake patients wear surgical masks (on top of supplementary oxygen, if required) 
en-route from ward/ED. In cases where a patient is not able to tolerate a surgical mask, the portering team will provide oxygen via oxygen mask and cover the transfer bed with disposable plastic sheet to minimise contamination along the transfer route (see Figure 2). Security personnel will be activated to control traffic for transfer. Corridors and lifts used are decontaminated by our cleaning team immediately after transfer.

This set-up is done by our Department and can be easily replicated. Setup was jury-rigged with 4 acrylic arm support commonly used in OT and duct tapes and designed in a way to slide easily under a pillow or a spinal board (see Figure 3).

Table 3. Essential components of preparing OT for a patient with COVID-19.

\begin{tabular}{|c|c|}
\hline & - Minimum 2 Senior Anaesthetists, 2 Anaesthesia Nurses, 2 Surgeons, 2 Scrub nurses (Team) \\
\hline & PPE required: \\
\hline & - Anaesthesia personnel: PAPR + N95 masks + disposable gowns + Double gloves \\
\hline & - All other personnel: in OT-PAPR + N95 masks + disposable gowns in OT. \\
\hline & Outside OT-N95 + goggles + gowns + Double gloves \\
\hline \multirow[t]{20}{*}{ Personnel } & Standby personnel in DSOT bank: 1 Anaesthesia, 1 AU nurse, 1 Scrub nurse all gowned up as appropriate \\
\hline & - All to be in appropriate PPE attire prior to the patient's arrival. \\
\hline & - Team Huddle between Nursing, Surgeons and Anaesthesia personnel is required before patient's arrival: \\
\hline & - Surgical and Anaesthesia plan \\
\hline & - Equipment required \\
\hline & - Environment planning \\
\hline & - Anaesthesia Equipment \\
\hline & - Inside OT: \\
\hline & $\diamond$ Draeger Advance Anaesthesia Machine with HME filters at both patient and machine ends of circuit \\
\hline & $\diamond$ Transparent plastic wrapped-Philips Monitor \\
\hline & $\diamond$ Complete Airway Cart with suctioning \\
\hline & $\diamond$ Airway adjuncts and masks \\
\hline & $\diamond$ Video laryngoscope \\
\hline & $\begin{array}{l}\diamond \text { Fully stocked Anaesthetic drug trolley as per EOT including muscle relaxants, emergency drugs, syringes, } \\
\text { IV and IA cannulae }\end{array}$ \\
\hline & $\diamond 1$ litre Plasmalyte and Normal Saline fluid bags X 5 each \\
\hline & $\diamond$ Plastic Wrapped Ultrasound Machine \\
\hline & $\diamond$ Bair Hugger \\
\hline & $\diamond$ Stethoscope \\
\hline & - Outside OT: \\
\hline & $\diamond$ fully-equipped Emergency Trolley with Defibrillator \\
\hline \multirow[t]{16}{*}{ Equipment } & $\diamond$ Level 1 fluid infusor TM \\
\hline & $\diamond$ Fluid warmers \\
\hline & $\diamond$ Malignant Hyperthermia $(\mathrm{MH})$ kit \\
\hline & $\diamond$ Intralipid \\
\hline & - Surgical Equipment \\
\hline & - Instruments (Laparoscopic and Open) \\
\hline & - Diathermy \\
\hline & - Fluids \\
\hline & - Suctioning \\
\hline & - Miscellaneous \\
\hline & - 2 plastic wrapped portable computers \\
\hline & - Spare Pens, Paper and Anaesthetic Charts \\
\hline & - Spare N95, surgical masks with face shields, PAPR and filters \\
\hline & - Phone number of corridor phone to be clearly pasted on the phone in the OT \\
\hline & - Signage specifying route of transport, decontaminating OT area \\
\hline & - Visual posters on PPE donning/doffing procedures \\
\hline
\end{tabular}




\section{Continued}

Environment

Movement-Aim to minimise movement of personnel and equipment in and out of OT
Conduct of Anaesthesia
- Day Surgery OT (DSOT) complex cordoned off for COVID confirmed or suspected cases. The whole of DSOT is sealed up with plastic sheets. Neighbouring DSOT for doffing of PPE.

. Of Patients

- DSOT has a separate entrance away from MOT for transfer of COVID-19 case.

- The trolley of the patient is to be wrapped in plastic sheets, with the patient on the trolley, in the corridor outside MOT reception, before the patient is wheeled into MOT reception.

- Surgical mask is to be worn over a patient's oxygen nasal prongs/face mask.

- After surgery, the patient is to be recovered in OT before transporting back to the isolation ward/ICU.

. Of Personnel

- Surgeons and Scrub Nurses are to only enter the OT AFTER intubation.

- Anaesthesia Personnel are to stay with patients in OT till they fully recover in OT, before Porter takes over from outside OT.

- During CODE BLUE, standby personnel in appropriate PPE will enter via scrub room

- Of Equipment

- Intraoperatively, requests for items are to be made via phone to Runner Nurse outside OT. This Runner (circulating) Nurse then places the requested item in the scrub room, leaves the scrub room first before the personnel in OT retrieves the item.

- Requests for blood products are made in a similar manner. Clean personnel are to bring the blood product to MOT Reception and the circulating nurse will collect it from there.

- As far as possible, minimise aerosol-generating procedures e.g. face-mask ventilation, patient coughing/retching, awake FOB intubation, high-flow nasal cannula, BIPAP.

- RA is preferred over GA. Patients should wear a surgical face mask at all times if surgery is performed under RA. If a patient requires oxygen supplementation, the surgical mask is to be worn over the nasal cannula/face mask.

- ETT is preferred over an LMA for the better air seal.

- Using a video laryngoscope to intubate is recommended for better vision of the glottis when wearing the PAPR, and to increase the distance between the intubator and patient's airway.

- Minimise circuit disconnection. If this is unavoidable, ensure positive pressure ventilation is ceased, turn the APL valve to zero, and clamp the endotracheal tube prior to disconnection. This technique may also be used before switching a patient from the transport ventilator to the anaesthetic machine. The patient should be preoxygenated and the duration of disconnection should be kept to a minimum to avoid exacerbating hypoxia in critically ill COVID-19 patients with respiratory failure.

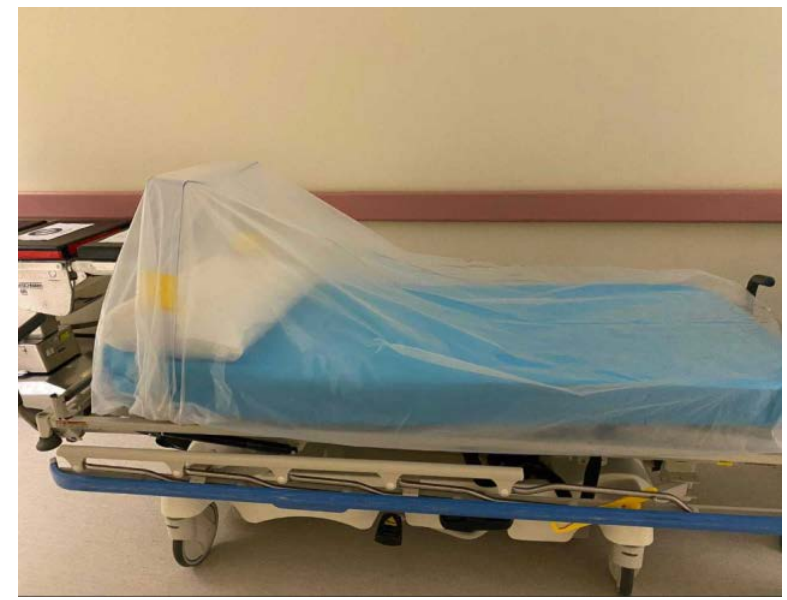

Figure 2. Transport trolley with transparent disposible plastic sheet to prevent aerosol/droplet contamination enroute to theatres. 


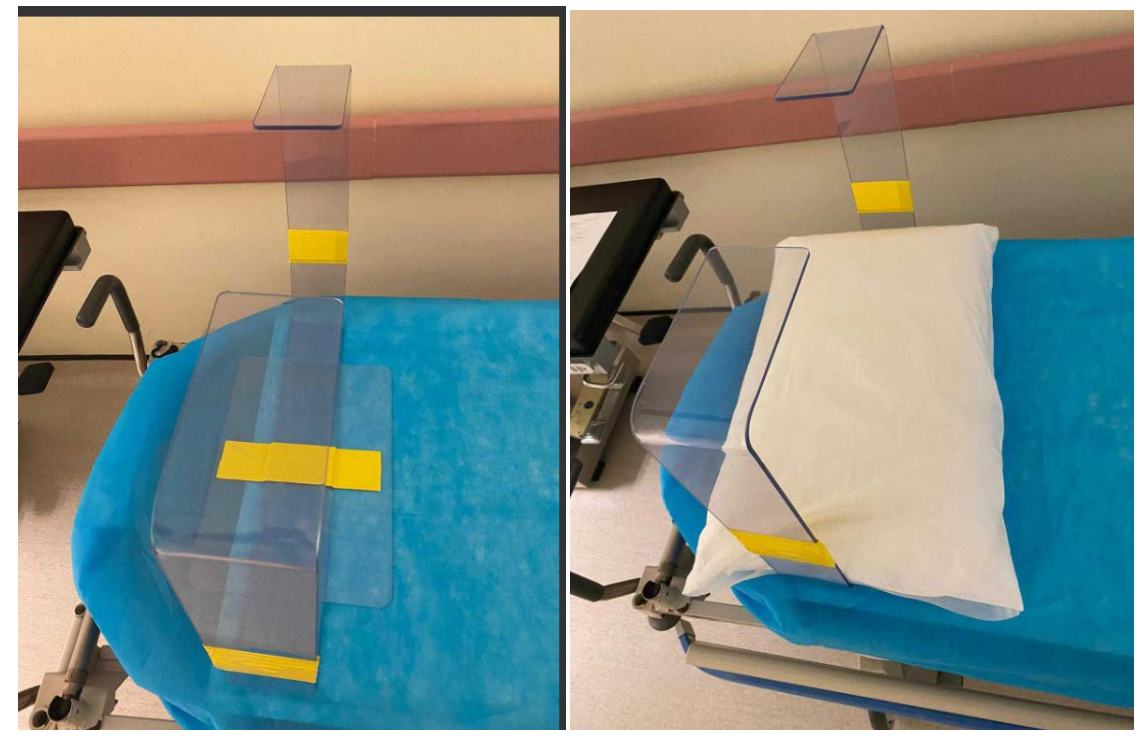

Figure 3. Fibreglass supports to improve patient comfort whilst under the transparent disposible plastic sheets enroute to theatre.

Signages are placed at strategic locations to alert staff of an ongoing case. The doors to the DSOT wing are closed immediately after patient transfer into the OT with clear signages barring entry and manual opening of the door to reduce airflow cross contamination. All communications with the team in DSOT are conducted via telecommunications through the assigned runners. Additional Equipment support is supplied in a manner to minimise contact of teams via support corridors.

\section{Personal Protective Equipment}

PPE is vital to protect the HCWs from exposure and being infected by patients with COVID-19. Worldwide shortage of PPE has been well documented. The supply chain team plays a very important role to ensure continuity of supply of PPE. To control and regulate this resource for the prolonged pandemic, a few measures were put in place.

From the time of the first recorded COVID-19 case in Singapore, OT ramped up stores of PPE by 10 times, based on our previous experiences during SARS and H1N1. However, stock depleted faster than expected, and to address this issue, a centralised control model was initiated institution-wide. Stocks were issued to allocated individuals at each departmental level. Individual departments were tasked to report directly to the deputy chairman of the medical board overseeing their division. This allowed for visibility and projection of needs, preventing and deterring mis-use and enabling us to ration our stocks of PPE in a sustained manner. Institution PPE needs were supplemented and supported by the Ministry of Health who also took active steps to procure more PPE for the frontline HCWs. In addition, our department started exploring other options such as CleanSpace ${ }^{\mathrm{TM}}$ Halo PAPR devices to supplement further PPE needs.

We recognised the need for prompt and correct donning of PPE to offer the best protection and allow for rapid response to any medical crisis such as that il- 
lustrated in the case vignette. PAPR and PPE refresher training started as early as December 2019 and allowed for rapid and confident deployment of medical care teams during any emergencies. Coordinated in-situ simulation in full PPE and PAPR were also conducted to enhance our readiness and look for systemic latent deficiencies.

Although our institution has generic guidelines regarding the level of PPE required for various outbreak scenarios. The emergence of the new SARS-CoV-2 virus with unknown transmission characteristics posed a challenge especially in the OT environment. Through frequent discussions with our inhouse infectious disease experts and review of new evidence and guidelines, our department came to a consensus on the use of PPE as well as tailored techniques during anaesthesia and critical care to help decrease accidental contamination of the environment and infection to HCWs.

\section{Decontamination procedure}

All equipment used in COVID-19 patients are deemed biohazard. In our case vignette, disposable surgical and anaesthetic equipment were utilised as possible and disposed as per the institutional protocol. Non-disposable instruments were sent directly to the Central Sterile Supply Department (CSSD) in a designated Biohazard cart. CSSD was informed in advance of such instances and their personnel were advised to wear full PPE when handling and decontaminating the equipment.

Environmental cleaning was performed by OT Assistants in PPE after the case as prescribed by our policies with particular attention paid to waste disposal. As the transmission route of COVID-19 was unclear at the point of our illustrated case, all waste from the OT was double bagged in biohazard bags and disposed into special sanitary sewer for treatment as per local sewerage disposal requirements. This has since been regularised for all the patients with/suspected SARS-CoV-2 infection.

\section{Death in OT area}

All personnel handling remains are instructed to wear full fluid repellant PPE. The body will be double-bagged before being taken out of the isolation room/premises. Biohazard labels are to be affixed onto the body bags. In the event that there are no laboratory tests conducted on the patients prior to the death, attending physicians would have to take post-mortem samples (2 sets of nasal and throat swabs or blood or tissues, as appropriate) before double bagging the deceased. The swab samples will be sent to the Microbiology laboratory locally and nationally. If the results come back positive, all attending staff perform self-temperature monitoring for 14 days and be on "Stay Home notice".

\subsection{Psychological Support of Healthcare Staff}

The uncertain climate, rapidly evolving situations and unfamiliar workflows inevitably cause a level of stress in any team. A recent study published by our colleagues in China has shown that the incidence of anxiety and stress disorder is high among medical staff working in these uncertain times [15]. 
Need for clear directives and flow of information is essential to allay anxieties. Peer support amongst colleagues is beneficial. To this end, our institution has available hotlines for emotional support during these challenging times. Staff can choose to utilise the hotline to make connections for peer support or for counselling support.

Our team reported increased fatigue on running our contingency roster for 3 weeks. Decision was made to switch back to our routine roster with a shadow roster system available such that implementation of contingency roster could take place almost immediately. Staff were also allowed rest days where they would remain contactable and easily activated should the need arise.

\section{Conclusion}

The COVID-19 pandemic is rapidly evolving on a global scale and is likely to have a protracted course. While guidelines in managing COVID-19 patients for Anaesthesia are useful, resource limitations and staff fatigue are challenges that must be addressed. We believe that all Anaesthesia units must take a proactive and practical approach to prepare for these potential challenges.

\section{Prior Presentation}

Not applicable.

\section{Funding}

None.

\section{Conflicts of Interest}

The authors declare no conflicts of interest regarding the publication of this paper.

\section{References}

[1] https://www.who.int/emergencies/diseases/novel-coronavirus-2019

[2] Wang, C., Horby, P., Hayden, F. and Gao, G. (2020) A Novel Coronavirus Outbreak of Global Health Concern. The Lancet, 395, 470-473. https://doi.org/10.1016/S0140-6736(20)30185-9

[3] Johns Hopkins University \& Medicine Coronavirus Resource Centre.

[4] MOH|Updates on COVID-19 (Coronavirus Disease 2019) Local Situation.

[5] Coronavirus (COVID-19) Events as They Happen.

[6] Wax, R.S. and Christian, M.D. (2020) Practical Recommendations for Critical Care and Anesthesiology Teams Caring for Novel Coronavirus (2019-nCoV) Patients. Canadian Journal of Anesthesia, 67, 568-576. https://doi.org/10.1007/s12630-020-01591-x

[7] https://www.moh.gov.sg/docs/librariesprovider5/diseases-updates/interim-pandemi c-plan-public-ver-_april-2014.pdf

[8] https://icmanaesthesiacovid-19.org

[9] Anaesthesia and Caring for Patients during the COVID-19 Outbreak. 
[10] https://www.cas.ca/en/practice-resources/news/cas-articles/2020/covid-19-recomme ndations-during-airway-manipulation

[11] Perioperative Considerations for the 2019 Novel Coronavirus (COVID-19).

[12] Frerk, C., Mitchell, V.S., McNarry, A.F., Mendonca, C., Bhagrath, R., Patel, A., O'Sullivan, E.P., Woodall, N.M. and Ahmad, I. (2015) Difficult Airway Society 2015 Guidelines for Management of Unanticipated Difficult Intubation in Adults. BJA: British Journal of Anaesthesia, 115, 827-848. https://doi.org/10.1093/bja/aev371

[13] Loh, N.W., Tan, Y., Taculod, J., et al. (2020) The Impact of High-Flow Nasal Cannula (HFNC) on Coughing Distance: Implications on Its Use during the Novel Coronavirus Disease Outbreak. Canadian Journal of Anesthesia, 67, 893-894. https://doi.org/10.1007/s12630-020-01634-3

[14] Ng, K., Poon, B.H., Kiat Puar, T.H., et al. (2020) COVID-19 and the Risk to Health Care Workers: A Case Report. Annals of Internal Medicine, 172, 766-767. https://doi.org/10.7326/L20-0175

[15] Huang, J.X., Han, M.F., Luo, T.D., et al. (2020) Survey of Mental Health of Medical Staff in Hospitals Receiving New Coronavirus Pneumonia. Chinese Journal of Occupational Diseases and Occupational Diseases. 